\title{
Challenges in Treating Patients with Major Depressive Disorder: The Impact of Biological and Social Factors
}

\author{
Soichiro Sato - Tzung Lieh Yeh
}

Published online: 28 May 2013

(C) Springer International Publishing Switzerland 2013

\begin{abstract}
Major depressive disorder (MDD) is frequently unrecognized and underdiagnosed by clinicians and thus remains untreated or inappropriately treated in routine clinical practice. Although the symptoms of MDD are widely acknowledged and recognized by clinicians, numerous epidemiological studies have reported that this disorder is more prevalent than had previously been thought, and that it is challenging to diagnose and treat, particularly because somatic symptoms and comorbid conditions are common in real clinical situations. MDD is associated with increased morbidity and mortality as well as with higher healthcare costs and more severe functional impairment. Therefore, optimal treatment for MDD should include collaboration focussed on comorbid physical diseases, rehabilitation aimed at restoring social functioning, and pharmacotherapy designed to ensure complete remission including psychological and physical symptoms, as well as functional recovery.
\end{abstract}

Previous Presentation: Part of the information presented in this article was presented at the round table discussion of psychiatrists from Asia and the USA, sponsored by Korea Otsuka International Asia and Arab.

\section{S. Sato $(\square)$}

Zikei Hospital/Zikei Institute of Psychiatry, 100-2

Urayasuhonmachi, Southern Ward, Okayama City,

Okayama, Japan

e-mail: soichiro@ja2.so-net.ne.jp

\section{T. L. Yeh}

Department of Psychiatry, National Cheng Kung University Hospital, College of Medicine, National Cheng Kung University, Tainan, Taiwan

\section{Introduction}

Major depressive disorder (MDD) is so common a mental illness that almost all physicians, irrespective of specialty and location, will see patients with this condition. Indeed, unipolar major depression is currently the fourth leading contributor to the global disease burden. According to the Global Burden of Disease Study, unipolar major depression will be ranked as the second leading cause of disabilityadjusted life-years, after ischaemic heart disease, in 2020. In 2030 , it is likely to be the leading cause of disabilityadjusted life-years [1, 2].

According to the nationwide survey conducted during 2001-2003, the lifetime prevalence of MDD is $16.6 \%$, and its 12-month prevalence is $6.7 \%$ in the USA [3]. In addition, a worldwide survey conducted during 2001-2003 by the World Health Organization found that the 12-month prevalence of mood disorders in the USA is $9.6 \%$ and $4.2 \%$ in Europe but only $2.6 \%$ in Korea, $3.1 \%$ in Japan, $2.5 \%$ in Beijing and $1.7 \%$ in Shanghai [4].

According to a survey conducted by the Ministry of Health, Welfare and Labour in Japan, the number of patients with mood disorders, including MDD and bipolar disorder, has increased from 433,000 in 1996 to 1,041,000 in 2008 [5]. This phenomenon reflects several changes in Japanese psychiatric care, including the shift from traditional observation-based diagnosis to the use of international classifications, such as the Diagnostic and Statistical Manual of Mental Disorders (DSM) or the International Classification of Diseases and Related Health Problems (ICD). The increasing familiarity of Japanese individuals with the term 'depression' has led to less hesitance among some people, especially those who are younger, in visiting a mental health clinic to address stress at work or school. Although the statistics may include some patients with 
mood disorders other than MDD, the number of patients diagnosed with MDD in clinical settings has certainly been increasing.

The prevalence of MDD in east Asian countries has long been underestimated. On the other hand, the suicide rate in east Asian countries, such as South Korea and Japan, has been higher than that in other countries [4]. These phenomena may be related to the cultural characteristics of this region. However, east Asian values have changed, and MDD must be treated appropriately to enable patients to live fulfilling lives. MDD treatment should not be limited to efforts to improve patients' symptoms but should also focus on patients' dignity and contribution to society.

According to some research, 30-50\% of MDD patients have severe depression, and more than $60 \%$ show severe role impairment $[3,6,7]$. In addition, sequenced treatment alternatives to relieve depression (STAR*D) trials demonstrated that approximately $30 \%$ of patients with MDD do not achieve remission after four antidepressant treatments but continue to experience significant impairment [8]. Therefore, approximately $30 \%$ of MDD patients seem to have treatment-resistant depression.

Risk factors for treatment-resistant depression include comorbid psychiatric and physical conditions, younger age at onset, severe depression at baseline, inadequate dose and duration of treatment, socioeconomic issues and suicidal ideation [8]. Therefore, both biological and social factors are involved in treatment-resistant depression.

\section{Biological Factors}

\subsection{Physical Symptoms in Major Depressive Disorder}

Although MDD is classified as a mood disorder, physical symptoms are common in patients with the DSM-IV [9] diagnosis of MDD. DSM-IV specifies nine criteria, including five psychological and four physical symptoms, for MDD. Insomnia, appetite and weight change, psychomotor retardation/agitation and lethargy/fatigue constitute the physical symptoms, and these can be very important indicators of depression. Physical symptoms may be either the cause or the consequence of depression.

According to Nakao et al. [10], the majority of depressed patients complain about some kind of physical symptoms, such as fatigue, nausea, pain, and so on. The rates of fatigue, insomnia and nausea among patients with depression have been reported to be $86 \%, 79 \%$, and $51 \%$, respectively (Table 1) [10]. The importance of physical symptoms in MDD was also replicated in a recent study that was conducted China, in which physical symptoms were highly associated with MDD and anxiety, regardless of the presence of underlying medical diseases [11].
Indeed, physical symptoms are very common among patients with MDD.

It was also reported that patients with MDD experience physical symptoms such as fatigue and loss of energy [12]. Indeed, approximately $90 \%$ of patients reported physical symptoms [13]. Physical symptoms are not related to the severity of MDD. In general, changes in physical symptoms are the first changes of which patients with MDD are aware. These somatic symptoms are so varied that patients may consult primary physicians rather than psychiatrists for help. However, physical examinations may show few remarkable results, suggesting that the symptoms may be related to MDD. This indirect route of help-seeking may delay both referral to psychiatric clinics and the diagnosis and treatment of MDD; these delays can cause poor treatment outcomes. Indeed, it has reported that approximately $30 \%$ of patients with MDD experience physical symptoms for more than 5 years before receiving the proper diagnosis [14-16].

Most depressed patients who fail to achieve remission have residual physical and psychological symptoms and the outcomes of patients with and without residual symptoms differ [16]. One study found that patients with residual symptoms had a three times higher risk of relapse compared with patients without residual symptoms [17]. More than $10 \%$ of patients who complain about one to three physical symptoms are likely to have a mood disorder. In addition, as the number of nonspecific physical symptoms increases, the likelihood of having a mood disorder also increases [13].

Table 1 Proportion of somatic symptoms in patients with depression

\begin{tabular}{ll}
\hline Symptoms & $\begin{array}{l}\text { Depressed patients reporting } \\
\text { symptom }(\%)\end{array}$ \\
\hline Fatigue & 86 \\
Insomnia & 79 \\
Nausea & 51 \\
Dyspnoea & 38 \\
Palpitations & 38 \\
Back pain & 36 \\
Diarrhoea & 29 \\
Headache & 28 \\
Chest pain & 27 \\
Sexual dysfunction & 23 \\
Pain in extremities & 20 \\
Dizziness & 19 \\
Abdominal pain & 18 \\
Tinnitus & 18 \\
Joint or limb pain & 16 \\
\hline
\end{tabular}

Adapted from Nakao et al. [10] 
Physical symptoms may also influence treatment response. In particular somatic symptoms do not adequately respond to selective serotonin reuptake inhibitors (SSRIs). However, serotonin and norepinephrine reuptake inhibitors (SNRIs) may have differential efficacy in MDD with physical symptoms compared to SSRIs because they inhibit both serotonin and norepinephrine, which are major neurotransmitters in pain pathways. In fact, SNRIs are approved for the treatment of pain-related diseases other than MDD itself $[18,19]$. Therefore, we may need clinical trial data with SNRIs for the control of physical symptoms because SNRIs may cause further improvement from partial response with SSRIs in MDD patients with physical symptoms [20]. A recent naturalistic, randomized trial found that treatment with SSRIs was associated with different effects as a function of the presence of physical symptoms; emotional symptoms were three times more likely to respond to this treatment than were physical symptoms [21]. After 9 months of SSRI treatment, emotional symptoms improved first, and feelings of positive wellbeing followed. Physical symptoms improved later, with non painful somatic symptoms improving third and painful somatic symptoms improving last. Therefore, the study indicates a possible relationship of physical symptoms and treatment outcomes, which should be more intensively studied in adequately powered and well-controlled clinical trials [21].

\subsection{Comorbid Physical Diseases}

MDD is considered an important risk factor for physical diseases. According to the STAR*D study of 1,500 patients, the total prevalence of physical diseases was $53 \%$. Patients who were older, had lower incomes, were unemployed, were less educated and had had depression for a longer duration were at higher risk of developing various medical disease. Musculoskeletal diseases, found in $43 \%$ of patients with MDD, were especially prevalent (Table 2) [22].

Table 2 Prevalence of comorbid physical diseases in patients with depression

\begin{tabular}{ll}
\hline Disease/system & Prevalence (\%) \\
\hline Eyes, ears, nose, throat and larynx & 45 \\
Musculoskeletal & 43 \\
Respiratory & 32 \\
Neurological & 27 \\
Upper gastrointestinal & 26 \\
Genitourinary & 25 \\
Endocrine & 24 \\
\hline
\end{tabular}

Adapted from Perahia et al. [20]

\subsection{Myocardial Infarction}

According to a naturalistic study including 896 post-myocardial infarction (MI) patients, the level of depression symptoms during admission for MI was found to be more closely associated with long-term survival than the level at 1 year, particularly in patients with moderate to severe levels of MDD, indicating that that the presumed cardiovascular mechanisms linking MDD to cardiac mortality may be more or less permanent for them [23]. Depression is a strong independent risk factor for ischaemic heart disease, and it alters platelet physiology in a way that may increase the risk of thrombus formation. Depression has a negative prognostic effect on mortality after acute MI. Follow-up of patients with MI more than 6 years after discharge indicated that the severity of depression was predictive of mortality and was positively associated with mortality [23].

\subsection{Diabetes Mellitus}

A meta-analysis of 42 studies showed that the risk of comorbid MDD in patients with diabetes was twice as high as that for people without diabetes [24]. MDD that is comorbid with diabetes tends to be more severe, chronic and prone to recurrence. Moreover, those with diabetes and comorbid depression are less likely to follow treatment recommendations (e.g. dietary restrictions, medication regimens, and blood glucose monitoring), which leads to poor outcomes. Depression is associated with a $60 \%$ increased risk of type 2 diabetes [24].

\subsection{Chronic Physical Diseases plus Major Depressive Disorder}

Patients with chronic physical diseases often have MDD. Indeed, the prevalence of MDD is higher in medically ill patients [25]. Comorbid MDD exacerbates patients' physical symptoms and decreases their pain threshold, which increases functional impairment. As a result, treatment adherence is reduced. Depression is also associated with adverse health behaviours and increased morbidity and mortality.

The presence of comorbid physical diseases is among the important risk factors for poor outcomes in MDD, and MDD is highly prevalent in patients with various medical conditions. Twenty-five per cent of patients with cancer, one-third of patients with Alzheimer's disease, and half of patients with Parkinson's disease have major depression, rendering MDD a potential cause of poor prognosis in such conditions [26, 27].

According to a global survey of 60 countries [28], the 12-month prevalence of MDD, angina and asthma was 
$3.2 \%, 4.5 \%$ and $3.3 \%$, respectively, in the absence of comorbid conditions. However, the average prevalence of comorbid depression in patients with one or more chronic physical disorders was between $9.3 \%$ and $23.0 \%$. This finding demonstrates that having a chronic physical disease is a significant risk factor for comorbid MDD. Moreover, having a chronic physical disease and comorbid MDD is more likely to lead to the worst outcomes related to the disease state than is having any chronic disease (including MDD) alone or than is having any other combination of chronic diseases that does not include MDD [28].

We must thus consider the possibility of comorbid MDD in patients with chronic physical diseases. We cannot expect better outcomes by treating MDD and physical diseases in isolation.

\section{Social Factors}

\subsection{Impairments in Physical and Social Functioning}

According to the National Comorbidity Survey Replication Study conducted by face-to-face household survey of 9090 respondents [6], most patients who have had MDD for 12 months experience some impairment in their social functioning as a result of this condition [6]. Impairments related to social roles were the most common such outcome
( $43.4 \%$ severe or very severe), and those related to work roles were the least common $(28.1 \%$ severe or very severe) [6]. Patients with MDD for 12 months reported a mean of 35.2 days of work missed during the past year because of MDD [6]. Indeed, both physical and social functioning are impaired by MDD. Moreover, social functioning is significantly more impaired by MDD than it is by other chronic illness such as hypertension, diabetes and arthritis [29]. The degree of impairment in the domains of work, home, relationships and/or other social functions is closely related to the severity of the MDD [6].

\subsection{Productivity and Economic Status}

In the USA, $7.2 \%$ of employees experience at least one depressive episode each year. Depressed individuals curtail their usual activities or stay in bed 66.4 days per year. It has been reported that depressed workers lose 27.2 work days per year and are responsible for a loss of US $\$ 36.6$ billion salary-equivalent productivity dollars per year; this group is also responsible for US\$27 billion in treatment costs [30]. Depressed workers report working at $70 \%$ of their peak productivity [30].

In Japan, the estimated total cost related to depressive disorders (ICD-10: F32.0-F33.9) was ¥3,090,050 million (approximately US $\$ 38.8$ billion) in 2008 . The direct cost was $¥ 209,036$ million (approximately US $\$ 2.63$ billion).
Fig. 1 Costs of major depressive disorder in Japan a proportion of costs; $\mathbf{b}$ absolute costs, unit: Japanese Yen. Adapted from Wells et al. [29] (a)

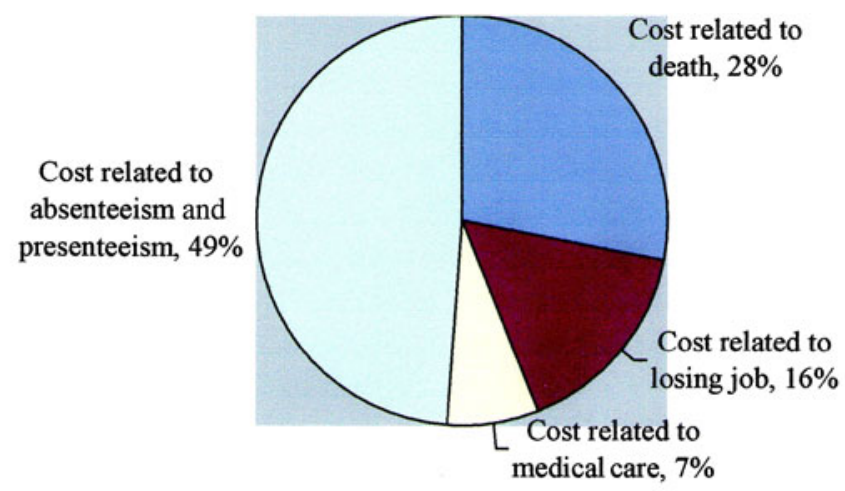

(b)

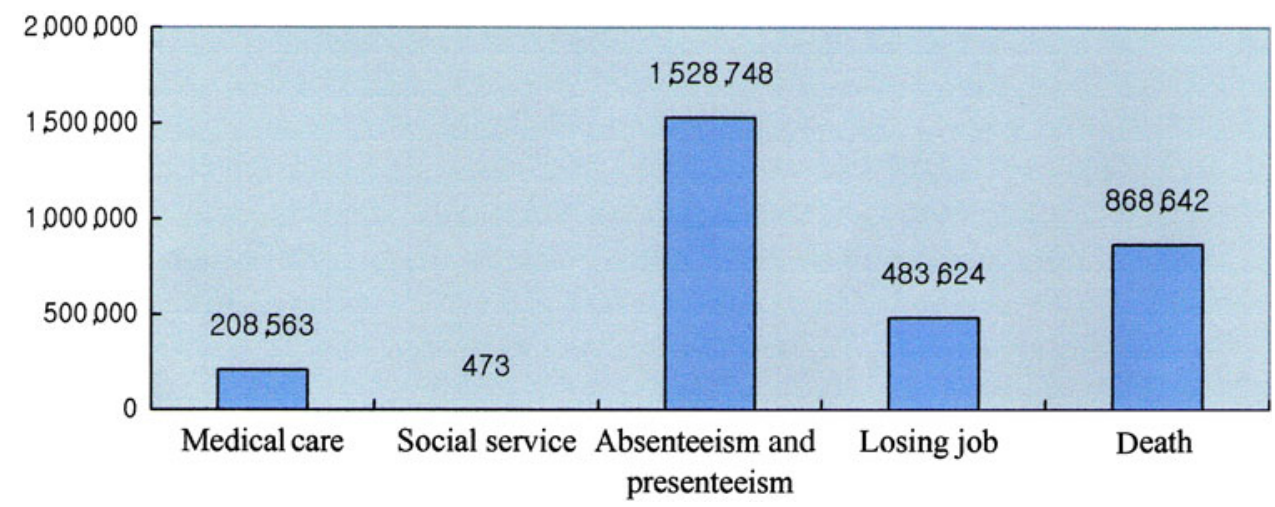


Considering absenteeism and presenteeism, an average of 68.7 days were lost to work, and the loss of productivity totalled $¥ 1,528,748$ million (US\$19.2 billion) (Fig. 1) [31].

MDD must be treated to restore the social and occupational functioning of patients with this disorder [32]. However, it is important that patients experience symptom remission, including improved cognition, before returning to work. Therefore, cognitive rehabilitation programmes are needed to enhance current treatment approaches to MDD.

Suicidality is also associated with MDD. Patients with major depression have a $2.2 \%$ lifetime risk of suicide compared with a less than $0.05 \%$ risk among those without this disorder, a 45-fold greater incidence [33].

\section{Conclusions and Future Directions}

Although MDD is common in east Asian countries, its prevalence has long been underestimated. One-third of patients with MDD meet the criteria for being considered treatment resistant. MDD is not only a mood disorder; it also entails physical disorders and disruption of social functioning. MDD exacerbates the physical symptoms associated with physical diseases, which may increase impairment in social functioning. Moreover, when comorbid with physical diseases, MDD can influence the outcomes of such diseases by leading to decreased treatment adherence and adverse health behaviours, which may increase mortality.

The treatment of physical symptoms increases the overall treatment response in MDD and the rate at which remission from this condition is achieved. Although MDD causes severe impairment in various domains of living, it is often difficult to identify these functional impairments in clinical settings. Each new episode of MDD tends to be more severe, longer, less responsive to treatment, and more likely to be followed by relapse as a result of a stressor. In addition, the interval between recurrences tends to become shorter [34].

Therefore, the optimal treatment for MDD should include collaboration focussed on comorbid physical diseases, rehabilitation aimed at restoring social functioning, and pharmacotherapy designed to ensure complete remission including psychological and physical symptoms as well as functional recovery.

Acknowledgments Although KOIAA was involved in supporting the production of the supplement, the content of the manuscript, its review and revision and the decision to submit to CNS Drugs were made solely by the authors and supplement guest editor.

Sources of Financial Support The authors did not receive honorarium for writing this paper.

Conflict of interest Drs Sato and Yeh have received honoraria from Korea Otsuka International Asia and Arab. The authors did not receive any payment for writing this manuscript.
Disclosure This manuscript has been published in a journal supplement that was created with an unrestricted educational grant from Korea Otsuka International Asia and Arab (KOIAA). The authors have received honoraria from KOIAA.

\section{References}

1. Murray CJ, Lopez AD. Evidence-based health policy-lessons from the Global Burden of Disease Study. Science. 1996;274: $740-3$.

2. Murray CJ, Lopez AD. The global burden of disease: a comprehensive assessment of mortality and disability from diseases, injuries, and risk factors in 1990 projected to 2020. Cambridge: Harvard School of Public Health; 1996.

3. Kessler RC, Chiu WT, Demler O, Merikangas KR, Walters EE. Prevalence, severity, and comorbidity of 12-month DSM-IV disorders in the National Comorbidity Survey Replication. Arch Gen Psychiatry. 2005;62:617-27.

4. Demyttenaere K, Bruffaerts R, Posada-Villa J, Gasquet I, Kovess $\mathrm{V}$, Lepine JP, et al. Prevalence, severity, and unmet need for treatment of mental disorders in the World Health Organization World Mental Health Surveys. JAMA. 2004;291:2581-90.

5. Ministry of Health, Welfare and Labour, Japan. National survey of injuries and diseases in Japan (in Japanese). http://www.e-stat. go.jp/SG1/estat/NewList.do?tid=000001031167. Accessed Aug 2012.

6. Kessler RC, Berglund P, Demler O, Jin R, Koretz D, Merikangas $\mathrm{KR}$, et al. The epidemiology of major depressive disorder: results from the National Comorbidity Survey Replication (NCS-R). JAMA. 2003;289:3095-105.

7. Kessler RC, Birnbaum H, Bromet E, Hwang I, Sampson N, Shahly V. Age differences in major depression: results from the National Comorbidity Survey Replication (NCS-R). Psychol Med. 2010;40:225-37.

8. Rush AJ, Warden D, Wisniewski SR, Fava M, Trivedi MH, Gaynes BN, et al. STAR*D: revising conventional wisdom. CNS Drugs. 2009;23:627-47.

9. Association AP. Diagnostic and statistical manual of mental disorders. 4th ed. Washington, DC: American Psychiatric Publishing; 1994.

10. Nakao M, Yamanaka G, Kuboki T. Major depression and somatic symptoms in a mind/body medicine clinic. Psychopathology. 2001;34:230-5.

11. Zhu C, Ou L, Geng Q, Zhang M, Ye R, Chen J, et al. Association of somatic symptoms with depression and anxiety in clinical patients of general hospitals in Guangzhou, China. Gen Hosp Psychiatry. 2012;34:113-20.

12. Kellner R, Sheffield BF. The one-week prevalence of symptoms in neurotic patients and normals. Am J Psychiatry. 1973;130: $102-5$.

13. Kroenke K, Spitzer RL, Williams JB, Linzer M, Hahn SR, deGruy FV, III, et al. Physical symptoms in primary care. Predictors of psychiatric disorders and functional impairment. Arch Fam Med. 1994;3:774-9.

14. Kroenke K, Bair MJ, Damush TM, Wu J, Hoke S, Sutherland J, et al. Optimized antidepressant therapy and pain self-management in primary care patients with depression and musculoskeletal pain: a randomized controlled trial. JAMA. 2009;301: 2099-110.

15. Kroenke K, Bair M, Damush T, Hoke S, Nicholas G, Kempf C, et al. Stepped Care for Affective Disorders and Musculoskeletal Pain (SCAMP) study: design and practical implications of an intervention for comorbid pain and depression. Gen Hosp Psychiatry. 2007;29:506-17. 
16. Tylee A, Gastpar M, Lepine JP, Mendlewicz J. Identification of depressed patient types in the community and their treatment needs: findings from the DEPRES II (Depression Research in European Society II) survey. DEPRES Steering Committee. Int Clin Psychopharmacol. 1999;14:153-65.

17. Paykel ES, Ramana R, Cooper Z, Hayhurst H, Kerr J, Barocka A. Residual symptoms after partial remission: an important outcome in depression. Psychol Med. 1995;25:1171-80.

18. Pae CU, Marks DM, Shah M, Han C, Ham BJ, Patkar AA, et al. Milnacipran: beyond a role of antidepressant. Clinical neuropharmacology. 2009;32:355-63.

19. Pae CU, Marks DC, Han C, Patkar AA, Masand PS. Duloxetine: an emerging evidence for fibromyalgia. Biomed Pharmacother. 2009;63:69-71.

20. Perahia DG, Quail D, Desaiah D, Montejo AL, Schatzberg AF. Switching to duloxetine in selective serotonin reuptake inhibitor non- and partial-responders: effects on painful physical symptoms of depression. J Psychiatr Res. 2009;43:512-8.

21. Greco T, Eckert G, Kroenke K. The outcome of physical symptoms with treatment of depression. $\mathrm{J}$ Gen Intern Med. 2004;19:813-8.

22. Yates WR, Mitchell J, Rush AJ, Trivedi MH, Wisniewski SR, Warden D, et al. Clinical features of depressed outpatients with and without co-occurring general medical conditions in STAR*D. Gen Hosp Psychiatry. 2004;26:421-9.

23. Lesperance F, Frasure-Smith N, Talajic M, Bourassa MG. Fiveyear risk of cardiac mortality in relation to initial severity and one-year changes in depression symptoms after myocardial infarction. Circulation. 2002;105:1049-53.

24. Mezuk B, Eaton WW, Albrecht S, Golden SH. Depression and type 2 diabetes over the lifespan: a meta-analysis. Diabetes Care. 2008;31:2383-90.
25. Sutor B, Rummans TA, Jowsey SG, Krahn LE, Martin MJ, O'Connor MK, et al. Major depression in medically ill patients. Mayo Clin Proc. 1998;73:329-37.

26. Baumeister H, Hutter N. Collaborative care for depression in medically ill patients. Curr Opin Psychiatry. 2012;25:405-14.

27. Katon WJ. Epidemiology and treatment of depression in patients with chronic medical illness. Dialogues Clin Neurosci. 2011;13:7-23.

28. Moussavi S, Chatterji S, Verdes E, Tandon A, Patel V, Ustun B. Depression, chronic diseases, and decrements in health: results from the World Health Surveys. Lancet. 2007;370:851-8.

29. Wells KB, Stewart A, Hays RD, Burnam MA, Rogers W, Daniels $\mathrm{M}$, et al. The functioning and well-being of depressed patients. Results from the Medical Outcomes Study. JAMA. 1989;262: 914-9.

30. Kessler RC, Akiskal HS, Ames M, Birnbaum H, Greenberg P, Hirschfeld RM, et al. Prevalence and effects of mood disorders on work performance in a nationally representative sample of U.S. workers. Am J Psychiatry. 2006;163:1561-8.

31. University K. Report on the estimate of the social cost of mental disorders (in Japanese). http://www.mhlw.go.jp/bunya/shougai hoken/cyousajigyou/d1/seikabutsu30-2.pdf. Accessed Aug 2012; 2011.

32. Miller IW, Keitner GI, Schatzberg AF, Klein DN, Thase ME, Rush AJ, et al. The treatment of chronic depression, part 3: psychosocial functioning before and after treatment with sertraline or imipramine. J Clin Psychiatry. 1998;59:608-19.

33. Bostwick JM, Pankratz VS. Affective disorders and suicide risk: a reexamination. Am J Psychiatry. 2000;157:1925-32.

34. Maixner SM, Greden JF. Extended antidepressant maintenance and discontinuation syndromes. Depress Anxiety. 1998;8(Suppl. 1): 43-53. 